\title{
Intrinsic hydrophilic character of carbon nanotube networks
}

\author{
Grzegorz Stando ${ }^{\mathrm{a}}$, Damian Łukawski ${ }^{\mathrm{b}}$, Filip Lisiecki ${ }^{\mathrm{c}}$, Dawid Janas ${ }^{\mathrm{a},{ }^{*}}$ \\ ${ }^{a}$ Department of Chemistry, Silesian University of Technology, B. Krzywoustego 4, 44-100 \\ Gliwice, Poland \\ ${ }^{b}$ Faculty of Technical Physics, Poznan University of Technology, Piotrowo 3, 60-965 \\ Poznan, Poland \\ ${ }^{c}$ Institute of Molecular Physics, Polish Academy of Sciences, Smoluchowskiego 17, 60-179 \\ Poznan, Poland
}

\begin{abstract}
We have demonstrated that nanocarbon materials such as carbon nanotubes (CNTs) or graphene have an intrinsically hydrophilic nature. Addition of liquid hydrocarbons or polycyclic aromatic compounds, which are the common side-products of nanocarbon syntheses, turned the otherwise hydrophilic CNT films hydrophobic. The very small amount needed to cause this effect was not discernable by SEM or Raman spectroscopy and also it could be easily overlooked by other methods of analysis. Upon simple and rapid thermal desorption of these species we were able to fully recover the water affinity in these CNT networks. Without using any oxidizing agents the material remains hydrophilic for weeks having water contact angle of about $35^{\circ}$. The results pave the way towards implementation of low-dimensional nanostructures in the real life without disrupting their inherent properties.
\end{abstract}

\section{Introduction}

Ever since carbon nanotubes (CNTs) and other forms of nanocarbon were discovered, they have attracted the attention of the R\&D community at many fronts most commonly even non related to each other such as optics [1-3], mechanical engineering [4, 5], electronics [6, 7], thermal management [8,9], etc. However, one of the major impediment factors that

\footnotetext{
${ }^{*}$ Corresponding author. Tel/Fax: +48 32 2371082. E-mail address: dawid.janas@polsl.pl (D. Janas).
} 
hampered the implementation of these materials in the real life is their poor compatibility with common everyday materials. Poor affinity towards hydrophilic media makes them extremely unsuitable for integration with polymer matrices without resorting to introduction of appropriate functional groups, which ensure proper interactions within the composite [10].

Recent results on graphene and graphite [11-14] reveal that there is an underlying reason why the nanocarbon may seem hydrophobic [15] or even superhydrophobic reaching water contact angles higher than $165^{\circ}[16]$. The effect is a result of hydrocarbon contamination introduced during the synthesis or airborne species present in the ambient $[11,13]$ deposit on the surface. It was shown that one can remove them by thermal annealing, ion bombardment or $\mathrm{UV} / \mathrm{O}_{3}$ treatment, but when exposed to air the surface becomes back hydrophobic (the water contact angle of $70-80^{\circ}$ ) within just 1 hour [11, 12]. Moreover, under certain conditions these treatments may affect the microstructure and composition of the material. Such defects may very much influence the observed water contact angles - in particular when measured in the static mode [14]. Deposition of water was found to slow down the process of surface character reversal by a factor of 20 by protecting the surface from hydrocarbon adsorption, but still the hydrophilicity could be preserved just for several hours [13].

In this contribution we give a strong indication that nanocarbon materials are intrinsically hydrophilic and can remain in this state for a very long time without any pretreatment steps when nanocarbon networks are prepared by a method recently developed by us $[17,18]$. By employing various hydrocarbon contaminants (model compounds of side-products introduced during synthesis) intentionally added into the CNT films we were able to study their influence on the water contact angle of the material. Addition of very low amounts of these species to the thermally annealed non-functionalized but hydrophilic CNT films made them hydrophobic. Conversely, thermal desorption of these compounds from the surface restored very good water wettability (ca $35^{\circ}$ contact angle), which was stable for weeks [18]. Our 
results not only give new insights into the surface nature of macroscopic ensembles from nanomaterials, but also demonstrate high potential for implementation of freshly-annealed materials in polymer matrices instead of subjecting them to destructive functionalization.

\section{Experimental}

Single-walled carbon nanotubes (SWCNTs) of high crystallinity were purchased from OCSiAl (Tuball ${ }^{\mathrm{TM}}$ ) and purified by air treatment and reflux in $\mathrm{HCl}$ according to a published routine [19]. Then, they were used to make CNT films by a method reported by us previously [17]. Nanocarbon material was combined with ethyl cellulose (EC) in acetone/toluene mixture. To ensure uniform dispersion, sonication was employed (Hielscher UP50H). Such CNT paint was subsequently deposited onto a Kapton foil, from which, once it has dried, it readily peeled off as a CNT-EC film. In the last step, EC was fully removed by igniting it with a lighter, which takes just a couple of seconds and does not affect the surface chemistry of the CNTs [18]. The whole process is illustrated in the Figure 1.

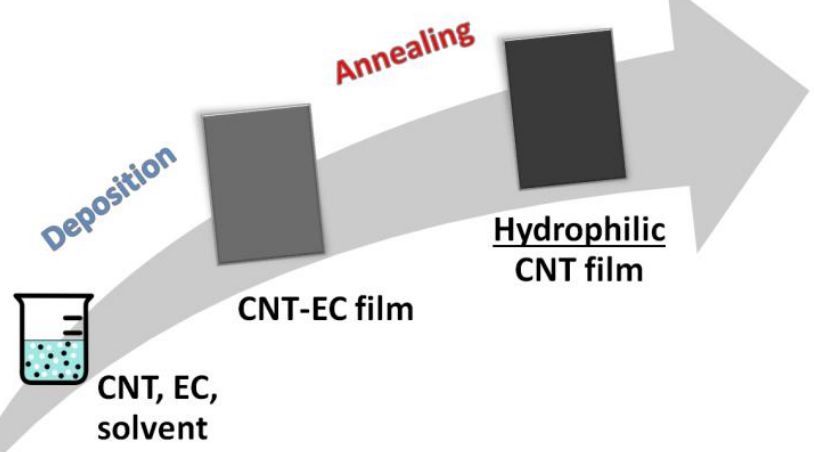

Fig. 1 Manufacture of free-standing hydrophilic CNT films.

Water contact angle (CA) measurements were carried out in the static mode using a custommade setup equipped with high-resolution macro camera. $20 \mu \mathrm{L}$ water droplets were deposited onto the surface of the film and the registered value of the contact angle was recorded. At least 10 droplets were measured for each parameter combination. 
In the case of liquid hydrocarbons (benzene, toluene, xylene), contact angle was measured after addition of $50 \mu \mathrm{L}$ of the respective compounds. Polyaromatic hydrocarbons (PAHs, naphthalene, phenanthrene and anthracene), graphite and carbon black were sonicated in acetone and applied in the same manner. The compounds were prepared in a range of concentrations: $3.33,10,25,60$ and $100 \mathrm{mg} / \mathrm{mL}$ concentrations (where possible).

Benzene (pure p.a.), toluene (pure p.a.), xylene (pure p.a.), acetone (pure p.a.), naphthalene (pure p.a.), phenanthrene (pure p.a.), anthracene (pure p.a.), graphite (flakes, p.a., 100 mesh) and were procured from Sigma-Aldrich. Carbon black (powder, p.a., average particle size 0.042 micron) was obtained from VWR.

1h waiting time was necessary between addition of hydrocarbon species and water contact angle measurement to enable complete evaporation of the liquid hydrocarbon or acetone. The results have shown that initially tested $24 \mathrm{~h}$ long break was excessive (Fig. S1).

Removal of added PAHs was carried out in the same way as EC was removed from the protofilm. Contact angle for these samples was measured after 14 days.

Scanning Electron Microscopy (SEM, FEI Nova NanoSEM 650) was used to probe the microstructure.

Raman spectroscopy (inVia Renishaw Raman microscope, $\lambda=633 \mathrm{~nm}$ ) detected inelastic scattering of the samples from 10 to $3200 \mathrm{~cm}^{-1} .25$ accumulations were acquired for each sample to diminish the effect of background noise. Mean values of calculated $\mathrm{I}_{\mathrm{D}} / \mathrm{I}_{\mathrm{G}}$ ratios and G peak maximum position are reported with corresponding standard deviations.

The roughness was measured by Zeiss LSM 510. The measurement and calculations were performed according to [20]. Briefly, topography map was obtained by LSM, using argon laser $(458 \mathrm{~nm})$ and EC Epiplan-Nefluar $20 \times$ lens. Then, the roughness was calculated by ZEN2011 software, as the arithmetic mean deviation of total surface height values RSa, according to DIN EN ISO 428. The topography map was taken for CNT and CNT-EC films, 
on 6 random spots for each sample ( 2 spots $\mathrm{x} 3 \mathrm{CNT}$ films) and the average value is presented in table below. The standard deviation of the mean was used as measurement uncertainty.

\section{Results and discussion}

\subsection{Contact angle measurements}

We started the evaluation of the character of the surface by comparing the water contact angle of the CNT films with and without EC (Fig. 2a). As previously reported, thermal annealing gives a binder-free CNT film of high water affinity [18]. The water contact angle was reduced from $95 \pm 4^{\circ}$ down to $33 \pm 5^{\circ}$ without introducing excessive functionalization, which is most commonly employed to make the material hydrophilic. Because acetone was the carrier liquid for PAH compounds used in the study, we had to eliminate the possibility that it could influence the surface character itself. No obvious change was detected as the recorded contact angle $\left(36 \pm 6^{\circ}\right)$ was negligibly different from that of the CNT film, which was not exposed to acetone. Since surface roughness variation could also be the reason for change of the apparent contact angle [21], we analyzed the surface of CNT films with and without EC by optical profilometer. The calculated surface roughness of these two materials was comparable $(6.4 \pm 1.9 \mu \mathrm{m}$ for CNT-EC film vs. $6.9 \pm 0.9 \mu \mathrm{m}$ for CNT film) we excluded this as an underlying reason for the observed effect. Recorded surface images are presented in Fig. S2. Next, we tested how liquid hydrocarbons in the form of benzene analogues (benzene, toluene, xylene) can influence the wettability of CNT films. This was to check if the observed unexpectedly high hydrophilic character of CNT films can be explained by the removal of hydrocarbon-based contaminants on the surface of CNTs [11, 13] introduced during the synthesis. Such compounds have been suspected to be responsible for the hydrophobic nature of nanocarbon materials, but experimental demonstration of this phenomenon was limited by the state-of-the-art. 
(a)

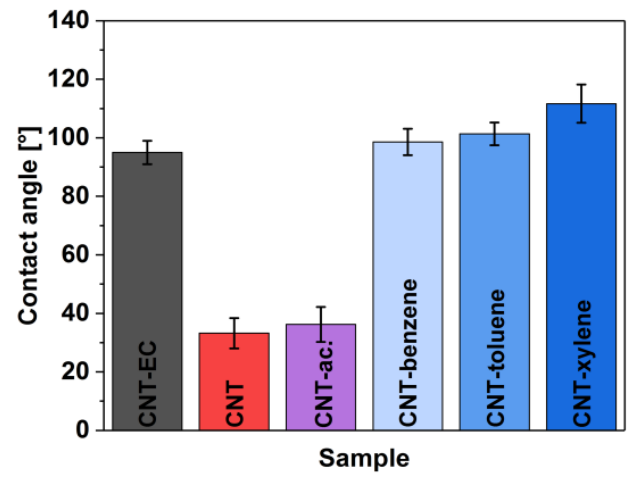

(b)

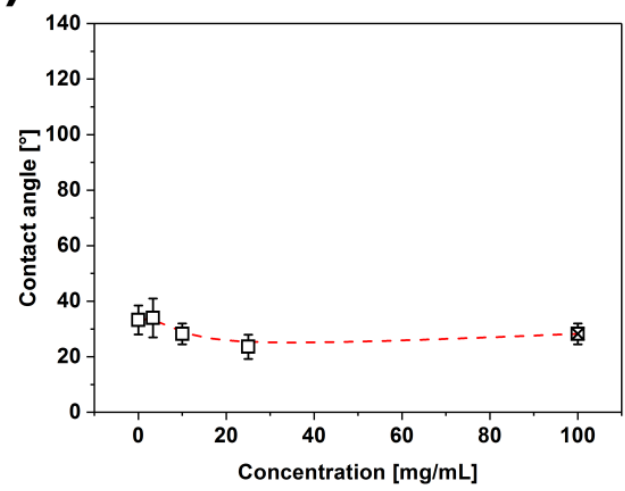

(c)

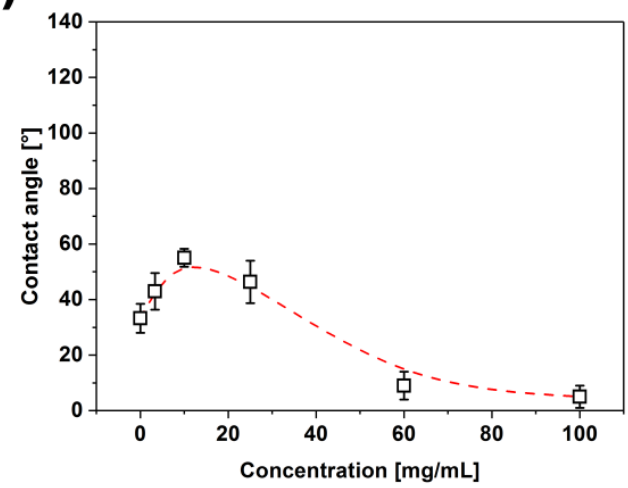

(d)

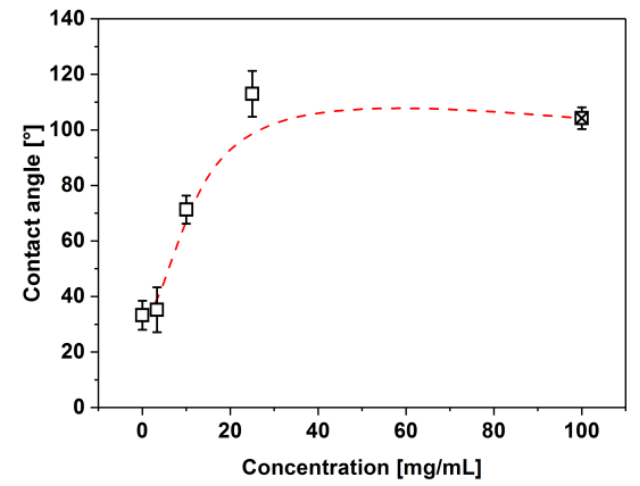

(e)

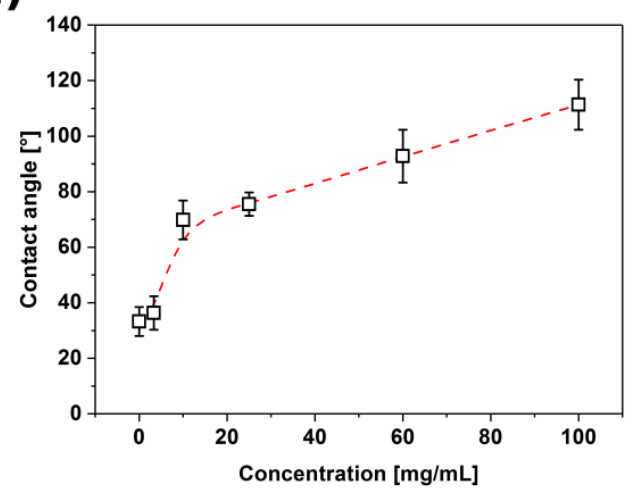

(f)

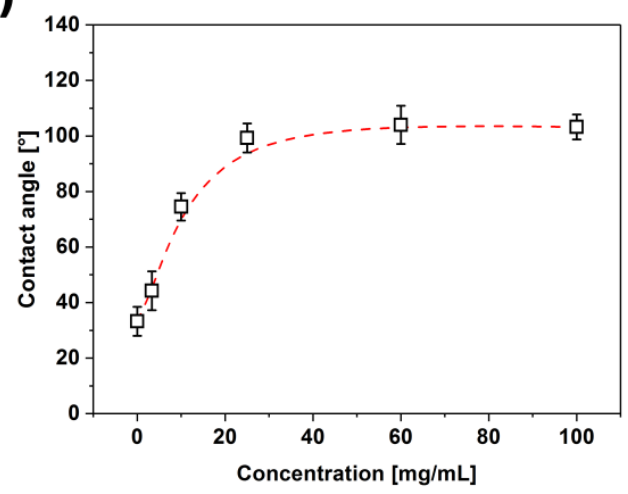

Fig. 2 Water contact angles of the samples (a) before removal of EC, after removal of EC, after removal of EC and addition of neat acetone, after addition of liquid hydrocarbons onto EC-free CNT films. Influence of concentration of PAH solution in acetone on the water contact angle using (b) carbon black, (c) graphite, (d) anthracene, (e) phenanthrene and (f) naphthalene.

Functionalization-free hydrophilic macroscopic ensembles from CNTs are a perfect model material to study this effect. As illustrated in Fig. 2a, addition of a small amount of benzene, toluene or xylene to the annealed hydrophilic films revives their hydrophobic nature. Contact 
angles in these cases reached $99 \pm 5^{\circ}, 101 \pm 4^{\circ}$ and $112 \pm 7^{\circ}$ respectively. The values are marginally higher than that of the CNT-EC film. That film contained the hydrocarbon contamination introduced during the synthesis, which justifies its poor wettability by water. This effect was counteracted to some extent by the presence of EC, which is more hydrophilic than nanocarbon materials (ca. $70^{\circ}$ as reported in our previous work [18]). The slight increase of water contact angle from benzene to xylene can be explained by their gradually decreasing affinity towards water as proven by water solubility and oil/water partition coefficients [22]. Importantly, the hydrophobic effect did not fade in time, which shows that such compounds can be strongly adsorbed on the surface by $\pi-\pi$ stacking and other interactions.

Although addition of small amount of liquid hydrocarbons is a convenient method to make the surface hydrophobic, using these compounds would be difficult to gauge how the hydrocarbon concentration affects the water contact angle. Volatile and neutral to contact angle acetone is a medium, in which these compounds are sparingly soluble, so we selected polyaromatic hydrocarbons (PAHs, naphthalene, phenanthrene and anthracene), graphite and carbon black as the candidate (macro)molecules to use them for this purpose. Carbon black, as a control material, which contains a range of chemisorbed oxygen moieties on the surface did not change the observed contact angle (Fig. 2b). The water contact angle was reduced slightly from $33 \pm 5^{\circ}$ to $24 \pm 4^{\circ}$ (recorded at $25 \mathrm{mg} / \mathrm{mL}$ concentration). Higher concentrations of the dispersions were highly non-uniform, therefore the $100 \mathrm{mg} / \mathrm{mL}$ value serves just as an indication of what was observed (indicated by a crossed rectangle in the plot). Graphite on the other hand showed an initial increase in the water contact angle peaking at $10 \mathrm{mg} / \mathrm{mL}$ $\left(55 \pm 3^{\circ}\right)$ and then tended to complete wetting $\left(5 \pm 4^{\circ}\right.$ at $\left.100 \mathrm{mg} / \mathrm{mL}\right)$. The hydrophobic character of graphite in the low concentration regime made the CNT film in some degree more hydrophobic. However, the effect was subsequently overpowered by the influence the 
graphite had on the microstructure of the material [23]. As indicated by SEM (described in details in the further parts of this contribution), graphite changes the surface roughness of the CNT film. The consequence is that we observe a very small water contact angle for CNT film, which is in agreement with Wenzel's theory that predicts such apparent hydrophilic character enhancement [24, 25]. Finally, PAH dispersions in acetone introduced to the surface of CNT films at various concentrations enabled us to probe their effect on material wettability. With increasing concentration of anthracene we observed a gradual increase of water contact angle (Fig. 2d). Even one droplet of anthracene dispersion in acetone at 10 $\mathrm{mg} / \mathrm{mL}$ was enough to increase the water contact angle from $33 \pm 5^{\circ}$ to $71 \pm 5^{\circ}$. The maximum water contact angle that we observed for this case was $113 \pm 8^{\circ}$ at $25 \mathrm{mg} / \mathrm{mL}$ of this PAH dispersion. Unfortunately, higher values were not possible to record as we have reached the solubility of anthracene in acetone. The water contact angle shown by a crossed rectangle for targeted $100 \mathrm{mg} / \mathrm{mL}$ concentration serves just as estimation because anthracene could not completely dissolve in this medium above $25 \mathrm{mg} / \mathrm{mL}$. As the following tested compound, we selected phenanthrene, which is soluble in acetone up to at least $100 \mathrm{mg} / \mathrm{mL}$. The results showed that with increase in its concentration, the water contact angle rose in a nonmonotonic fashion to about $100^{\circ}$ (Fig. 2e). Water contact angles at this level have been commonly reported for as-made isotropic CNT ensembles [26, 27]. The relation between water contact angle and the concentration of the added PAH compound was even more pronounced when naphthalene was employed. Addition of just one droplet of $25 \mathrm{mg} / \mathrm{mL}$ dispersion reversed the hydrophilic character of the surface to hydrophobic $\left(99 \pm 5^{\circ}\right)$. Further increase in naphthalene content did not cause any further noticeable change. Fig. 3 depicts how the hydrophilic character of pure CNT film faded with increasing naphthalene concentration. It is important to mention that the introduced amount of this particular PAH to give rise to this effect is almost negligible $(\sim 2 \%$ wt as related to the weight of neat CNT film 
for $25 \mathrm{mg} / \mathrm{mL}$ concentration of the naphthalene dispersion), hence it can be very easily overlooked in themograms when multiple thermally-driven desorption processes and oxidation reactions take place in parallel.

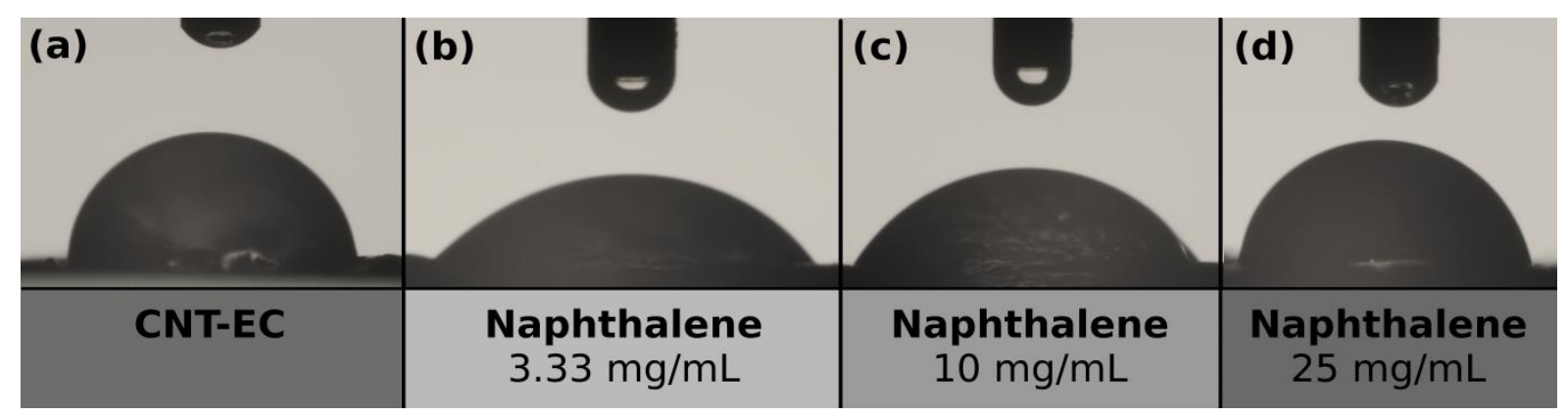

Fig. 3 Water contact angle images of the selected samples.

\subsection{Analysis of material microstructure and surface chemistry}

Microstructure of the material can often have a significant effect on the observed wettability of the surface, therefore we carried out SEM analysis of the material (Fig. 4). The proto-film before annealing, which contained EC, had evidently larger size of the CNT bundles (Fig. 4a). One can see that the bundles are thicker because EC being a good CNT dispersing agent [17] covers their surface. That also justifies why the observed water contact angle $\left(95 \pm 4^{\circ}\right)$ is lower than expected for as-made CNT ensembles. As stressed earlier in this contribution, EC is more hydrophilic and it decreases the observed water contact angle. Furthermore, the CNT film after annealing showed expected isotropic distribution of CNT bundles with minimal amount of non-CNT contaminants (Fig. 4b). What is very important, addition of acetone to the film does not cause capillary densification, which is very common for CNT ensembles (Fig. 4c) [28, 29] and may be actually unwanted for some applications. In our case it is a very important proof for the interpretation of results because it excludes the possible influence of solvent on the recorded wettability character of the material. 


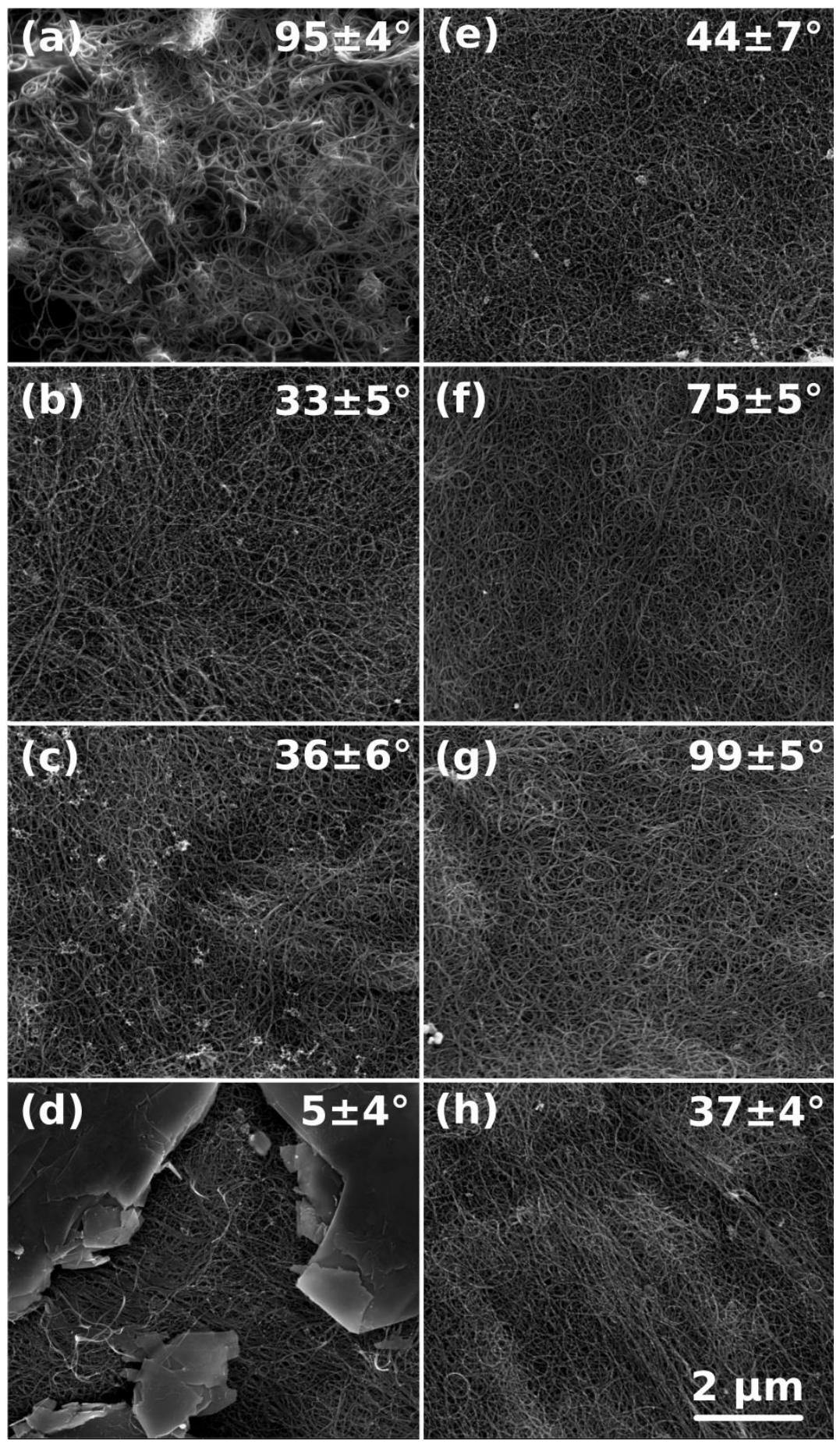

Fig. 4 SEM micrographs of films samples made of (a) CNT-EC, (b) CNT, (c) CNT after acetone treatment, (d) CNT after graphite dispersion treatment $(100 \mathrm{mg} / \mathrm{mL})$, (e) CNT after naphthalene dispersion treatment $(3.33 \mathrm{mg} / \mathrm{mL})$, (f) CNT after naphthalene dispersion treatment $(10 \mathrm{mg} / \mathrm{mL}),(\mathrm{g}) \mathrm{CNT}$ after naphthalene dispersion treatment $(25 \mathrm{mg} / \mathrm{mL}),(\mathrm{h}) \mathrm{CNT}$ after naphthalene dispersion treatment $(25 \mathrm{mg} / \mathrm{mL})$ with subsequent removal. 
Fig. 4d and Fig. S3 shows how such change in the microstructure can have a strong impact on the observed affinity towards water. The presence of graphite flakes led to the seemingly complete wetting of the material (Fig. 2c). What regards the naphthalene, its addition did not result in any evident changes to the microstructure of the material (Fig. 4e-g), so observed changes to the water contact angle with concentration are purely physical in nature. It is also important to mention that subsequent removal of naphthalene from the CNT film by annealing to recover the water affinity also does not result in any deterioration of the surface (Fig. 4h). This treatment reduced the water contact angle down to $37 \pm 4^{\circ}$. Despite radically different surface character (hydrophilic/hydrophobic) the microstructure of the material is indiscernible because low molecular weight naphthalene cannot be seen by this technique.
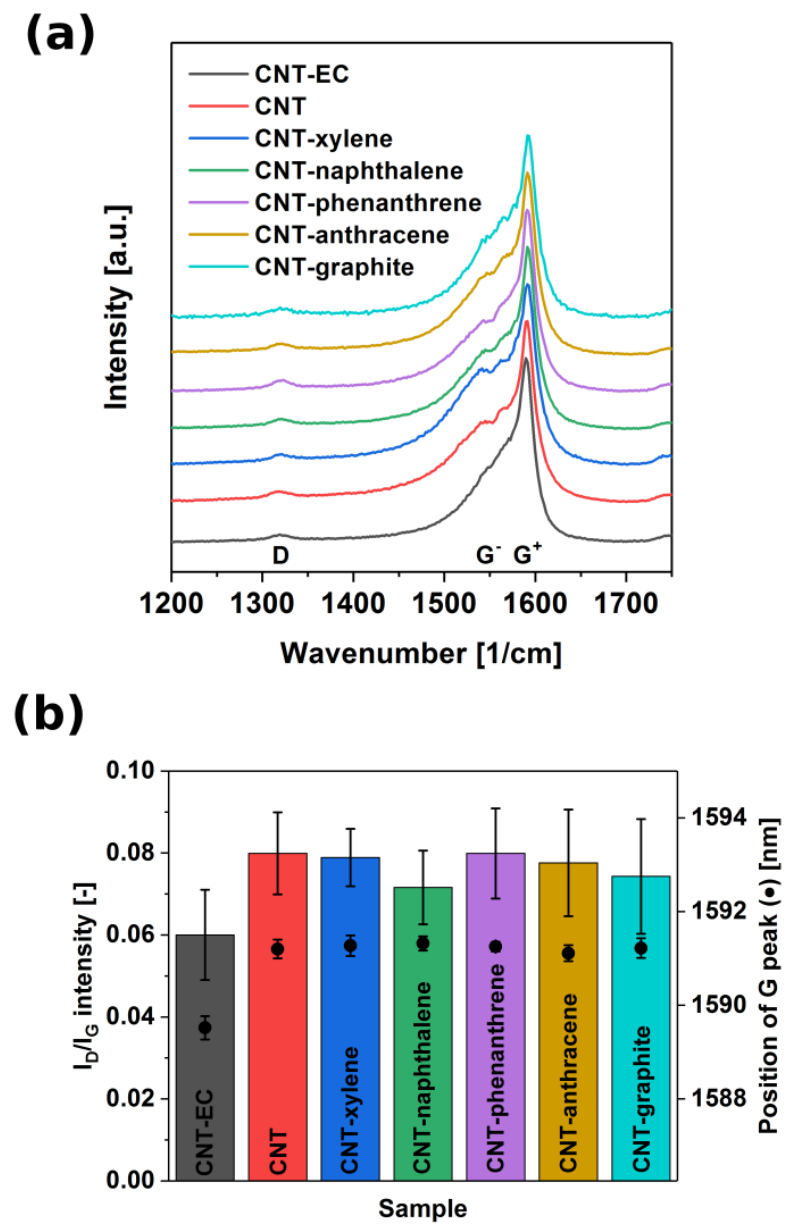

Fig. 5 (a) Raman spectra of selected samples, (b) $I_{D} / I_{G}$ intensity ratios and position of $G$ peak maximum of selected samples derived from corresponding Raman spectra. 
Next, we carried out characterization of the surface chemistry of the material by Raman spectroscopy (Fig. 5). Removal of EC from the CNT film by thermal annealing caused a slight increase of $\mathrm{I}_{\mathrm{D}} / \mathrm{I}_{\mathrm{G}}$ ratio from $0.06 \pm 0.01$ to $0.08 \pm 0.01$, but even so the material can still be considered as relatively pristine (as gauged by the low intensity of the disorder band D). Addition of liquid hydrocarbons, PAHs or graphite $(25 \mathrm{mg} / \mathrm{mL}$ concentration $)$ cannot be discerned in the Raman spectra. The $\mathrm{I}_{\mathrm{D}} / \mathrm{I}_{\mathrm{G}}$ ratios are very similar to that of the neat CNT film. Even though the presence of such impurities has a dramatic influence on the wettability of the surface, the content to cause this behavior is extremely low. Moreover, the position of the G peak shifted to higher wavenumbers after the removal of EC. After the annealing step, EC no longer wraps the CNTs and their bundles, which releases them from each other to some extent. Less intertube interactions has been reported to cause such change to the shape of the Raman spectra in CNTs [30]. No further notable change to the position the G peak could be recognized upon intentional addition of the contaminants, which indicates that neither significant changes to the CNT structure nor doping occur in this cases [31].

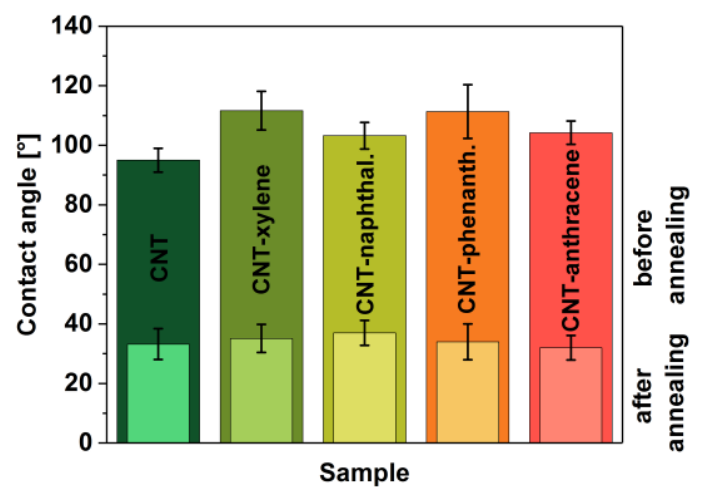

Fig. 6 Water contact angle of selected samples before and after annealing.

Finally, we wanted to show how the removal of the introduced compounds at $100 \mathrm{mg} / \mathrm{mL}$ concentration restores the hydrophilic character of the surface. In all of the cases we observed that the water contact angle returned to ca $35^{\circ}$, which very well matched that of the freshly 
annealed neat CNT film. This finding strongly suggests that nanocarbon materials such as graphene/CNTs may be inherently hydrophilic and only the presence of airborne hydrocarbon contaminants [11] deposited on their surface make them seemingly hydrophobic. Our model compounds, which are common side-products of CNT synthesis, showed that various PAHs are able to cause this effect to a very similar extent. CVD of CNTs can produce over 45 sideproducts including methane, volatile organic compounds (VOCs) and PAHs [32]. For the applications which require good wetting of the material with hydrophilic compounds, appropriate means of purification carried out immediately before use are necessary. This contribution puts forward a convenient solution, which satisfies these requirements.

\section{Conclusions}

We presented a strong proof that CNT networks are intrinsically hydrophilic. The reason for the commonly observed hydrophobic behaviour of nanocarbon material is caused by hydrocarbon contaminants, which are introduced into the material during the synthesis. No method of production of graphene, CNTs and other allotropes of carbon is $100 \%$ selective due to the underlying set of complex and intertwined chemical reactions. The developed by us method of manufacture of thin films from nanocarbon materials gives highly hydrophilic material. The reason for the observed behavior is the flash annealing step, which removes not only EC, but also the hydrocarbon contaminants present on the surface. The phenomenon was validated herein using a number of polycyclic aromatic hydrocarbons: anthracene, phenanthrene and naphthalene at different concentrations as well as liquid hydrocarbons.

What is very important, the intrinsic wetting nature can be maintained for much longer than in the contributions reported so far (days instead of hours without any pre-treatment to the material). The recovery of the water contact angle is most rapid at the beginning therefore integration of such CNT material with e.g. hydrophilic polymer matrices should be carried 
out immediately after the thermal desorption of the contaminating species. Since we do not introduce functional groups into the material to make it hydrophilic we are able to preserve the properties of the nanocarbon component. We believe that this technique could be very helpful for fabrication of high-performance devices and other macroscopic applications of CNTs and graphene. From the fundamental science point of view, we can clearly see that the presented findings add another dimension to the study of the tailorable nanocarbon surface. It is highly probable that the method developed by us, which gives thin free-standing films, can be extended to make other non-functionalized, but hydrophilic low-dimensional nanostructures, which we plan to explore in the upcoming future.

\section{Competing financial interests}

The authors declare that there is not any competing financial interest in relation to the work described.

\section{Acknowledgements}

D.J. and G.S. thank National Science Center, Poland (under the Polonez program, grant agreement UMO-2015/19/P/ST5/03799) and the European Union's Horizon 2020 research and innovation programme (Marie Skłodowska-Curie grant agreement 665778). D.J. would also like to acknowledge Foundation for Polish Science for START scholarship (START 025.2017), the Ministry for Science and Higher Education for the scholarship for oustanding young scientists (0388/E-367/STYP/12/2017) and the Rector of the Silesian University of Technology in Gliwice for the Pro-Quality grant (04/020/ RGJ18/0057). D.Ł. thanks National Science Center, Poland (under the Preludium program, grant agreement UMO2015/19/N/ST8/02184) 


\section{References}

[1] H. Kataura, Y. Kumazawa, Y. Maniwa, I. Umezu, S. Suzuki, Y. Ohtsuka, Y. Achiba, Optical properties of single-wall carbon nanotubes, Synthetic Metals 103(1) (1999) 2555-2558.

[2] S.M. Bachilo, M.S. Strano, C. Kittrell, R.H. Hauge, R.E. Smalley, R.B. Weisman, Structure-Assigned Optical Spectra of Single-Walled Carbon Nanotubes, Science 298(5602) (2002) 2361.

[3] D. Janas, N. Czechowski, B. Krajnik, S. Mackowski, K.K. Koziol, Electroluminescence from carbon nanotube films resistively heated in air, Applied Physics Letters 102(18) (2013) 181104.

[4] M.-F. Yu, O. Lourie, M.J. Dyer, K. Moloni, T.F. Kelly, R.S. Ruoff, Strength and Breaking Mechanism of Multiwalled Carbon Nanotubes Under Tensile Load, Science 287(5453) (2000) 637-640.

[5] F.H. Gojny, M.H.G. Wichmann, B. Fiedler, K. Schulte, Influence of different carbon nanotubes on the mechanical properties of epoxy matrix composites - A comparative study, Composites Science and Technology 65(15) (2005) 2300-2313.

[6] T.W. Odom, J.-L. Huang, P. Kim, C.M. Lieber, Atomic structure and electronic properties of singlewalled carbon nanotubes, Nature 391 (1998) 62.

[7] P.G. Collins, K. Bradley, M. Ishigami, A. Zettl, Extreme Oxygen Sensitivity of Electronic Properties of Carbon Nanotubes, Science 287(5459) (2000) 1801.

[8] S. Berber, Y.-K. Kwon, D. Tománek, Unusually High Thermal Conductivity of Carbon Nanotubes, Physical Review Letters 84(20) (2000) 4613-4616.

[9] R.S. Ruoff, D.C. Lorents, Mechanical and thermal properties of carbon nanotubes, Carbon 33(7) (1995) 925-930.

[10] P.-C. Ma, N.A. Siddiqui, G. Marom, J.-K. Kim, Dispersion and functionalization of carbon nanotubes for polymer-based nanocomposites: A review, Composites Part A: Applied Science and Manufacturing 41(10) (2010) 1345-1367.

[11] Z. Li, Y. Wang, A. Kozbial, G. Shenoy, F. Zhou, R. McGinley, P. Ireland, B. Morganstein, A. Kunkel, S.P. Surwade, L. Li, H. Liu, Effect of airborne contaminants on the wettability of supported graphene and graphite, Nature Materials 12 (2013) 925.

[12] A. Kozbial, Z. Li, J. Sun, X. Gong, F. Zhou, Y. Wang, H. Xu, H. Liu, L. Li, Understanding the intrinsic water wettability of graphite, Carbon 74 (2014) 218-225.

[13] Z. Li, A. Kozbial, N. Nioradze, D. Parobek, G.J. Shenoy, M. Salim, S. Amemiya, L. Li, H. Liu, Water Protects Graphitic Surface from Airborne Hydrocarbon Contamination, ACS Nano 10(1) (2016) 349359.

[14] A. Kozbial, C. Trouba, H. Liu, L. Li, Characterization of the Intrinsic Water Wettability of Graphite Using Contact Angle Measurements: Effect of Defects on Static and Dynamic Contact Angles, Langmuir 33(4) (2017) 959-967.

[15] M. Bottini, S. Bruckner, K. Nika, N. Bottini, S. Bellucci, A. Magrini, A. Bergamaschi, T. Mustelin, Multi-walled carbon nanotubes induce T lymphocyte apoptosis, Toxicology Letters 160(2) (2006) 121-126.

[16] C. Journet, S. Moulinet, C. Ybert, S.T. Purcell, L. Bocquet, Contact angle measurements on superhydrophobic carbon nanotube forests: Effect of fluid pressure, EPL (Europhysics Letters) 71(1) (2005) 104.

[17] D. Janas, M. Rdest, K.K.K. Koziol, Free-standing films from chirality-controlled carbon nanotubes, Materials \& Design 121 (2017) 119-125.

[18] D. Janas, G. Stando, Unexpectedly strong hydrophilic character of free-standing thin films from carbon nanotubes, Scientific Reports 7(1) (2017) 12274.

[19] A.J. Clancy, E.R. White, H.H. Tay, H.C. Yau, M.S.P. Shaffer, Systematic comparison of conventional and reductive single-walled carbon nanotube purifications, Carbon 108 (2016) 423-432.

[20] T. Bezák, M. Kusý, M. Eliáš, M. Kopček, Surface roughness determination using laser scanning confocal microscope Zeiss LSM 700, METAL 2013 - 22nd International Conference on Metallurgy and Materials, Brno, Czech Republic. 
[21] K.J. Kubiak, M.C.T. Wilson, T.G. Mathia, P. Carval, Wettability versus roughness of engineering surfaces, Wear 271(3) (2011) 523-528.

[22] A. Sato, T. Nakajima, Partition coefficients of some aromatic hydrocarbons and ketones in water, blood and oil, British Journal of Industrial Medicine 36(3) (1979) 231-234.

[23] Y. Yuan, R. Lee, Contact Angle and Wetting Properties, Springer Berlin Heidelberg2013.

[24] R.N. Wenzel, Surface Roughness and Contact Angle, The Journal of Physical and Colloid Chemistry 53(9) (1949) 1466-1467.

[25] D.M. Kalaskar, F. Alshomer, Chapter 8 - Micro- and Nanotopographical Cues Guiding Biomaterial Host Response A2 - Lee, Sang Jin, in: J.J. Yoo, A. Atala (Eds.), In Situ Tissue Regeneration, Academic Press, Boston, 2016, pp. 137-163.

[26] H. Liu, J. Zhai, L. Jiang, Wetting and anti-wetting on aligned carbon nanotube films, Soft Matter 2(10) (2006) 811-821.

[27] T. Werder, J.H. Walther, R.L. Jaffe, T. Halicioglu, P. Koumoutsakos, On the Water-Carbon Interaction for Use in Molecular Dynamics Simulations of Graphite and Carbon Nanotubes, The Journal of Physical Chemistry B 107(6) (2003) 1345-1352.

[28] F.L.D.V. Michaël, P. Sei Jin, H.T. Sameh, O.V. Daniel, A.J. Hart, Fabrication and electrical integration of robust carbon nanotube micropillars by self-directed elastocapillary densification, Journal of Micromechanics and Microengineering 21(4) (2011) 045033.

[29] D. Janas, A. Cabrero-Vilatela, J. Bulmer, L. Kurzepa, K.K. Koziol, Carbon nanotube wires for hightemperature performance, Carbon 64 (2013) 305-314.

[30] L. Bokobza, J. Zhang, Raman spectroscopic characterization of multiwall carbon nanotubes and of composites, Express Polymer Letters 6(7) (2012) 601 - 608.

[31] D. Janas, Powerful doping of chirality-sorted carbon nanotube films, Vacuum 149 (2018) 48-52.

[32] D.L. Plata, A.J. Hart, C.M. Reddy, P.M. Gschwend, Early Evaluation of Potential Environmental Impacts of Carbon Nanotube Synthesis by Chemical Vapor Deposition, Environmental Science \& Technology 43(21) (2009) 8367-8373. 